Original Article

\title{
In silico exploration the phenolic compound of olive leaves as acetylcholinesterase enzyme (AChE) inhibitor for Alzheimer's disease therapy
}

\author{
Nurul Jadid Mubarakati ${ }^{1 *}$, Oktavia Rahayu Puspitarini ${ }^{2}$, Tintrim Rahayu ${ }^{1}$, Alik Maulidiyah ${ }^{3}$ \\ ${ }^{1}$ Department of Biology, Faculty of Science and Mathematics, University Islamic of Malang, Malang, Indonesia \\ ${ }^{2}$ Faculty of Animal Husbandry, University Islamic of Malang, Malang, Indonesia \\ ${ }^{3}$ Faculty of Science and Mathematics, Diponegoro University, Semarang, Indonesia
}

\begin{abstract}
Olive (Olea europaea) have been cultivated and grown well in tropical climates such as Indonesia. Indonesia local community have used olive as herbal medicines due to its active compounds known as oleuropein that has many biological activities including as neurotherapy in Alzheimer's disease. The purpose of this study is to explore the potential phenolic compounds of olive and examine the acetylcholinesterase (AChE) inhibitory activity displayed by different olive polyphenols through a silico approach. The bioactive compounds of olive which had been analyzed in this study were phenolic compound included oleuropein, demethyl-oleuropein, ligstroside, oleoside, verbascoside, luteolin 7-glucoside, and hydroxytyrosol. Interaction of bioactive compounds with acetylcholinesterase (AChE) was analyzed through molecular specific docking using AutoDock Vina with Pyrx Software. The result elucidate that olive contain potential biological activities as antioxidant, anti-inflammatory, antineoplastic, free radical scavenger, antibacterial, antifungal, expression TP53 enhancer, caspase 8 stimulant, platelet adhesion inhibitor, treatment for lipoprotein disorder, antiviral and dementia treatment/Alzheimer disease. The highest bioactivity percentage of olive phenolic compound are as an antioxidant of $82 \%$, anti-inflammatory of $73 \%$, and anti-cancer (antineoplastic) of $70 \%$ respectively. Based on molecular docking analysis show one of olive phenolic compound of the dimethyl-oleuropein has strong interaction with AChE as pointed in the binding affinity of demethyl-oleuropein + AChE of -8.9 $\mathrm{kcal} / \mathrm{mol}$ has closed to galanthamine binding affinity of $-10.3 \mathrm{kcal} / \mathrm{mol}$. Along with glutamate acid 202 , tyrosine 133 and tyrosine 124 are the major contributors in the target-ligand interactions. The selected demethyl-oleuropein ought to be tested in clinical studies to discover new neuro-therapeutic candidates.
\end{abstract}

Keywords: Acetylcholinesterase enzyme (AChE) inhibitor, Alzheimer's disease, in silico approach, olive

Received: 14 December 2018 Revised: 16 May 2019 Accepted: 16 June 2019

\section{Introduction}

Alzheimer's (PA) is a disease caused by a progressive neurodegenerative disorder with many cognitive and neuropsychiatric symptoms in the elderly population. This disease is characterized by a decrease in acetylcholine (Ach) in the brain. Acetylcholine is a chemical messenger that is important about one's cognitive function (Konrath et al., 2013).

The deficiency of cholinergic neurotransmission activity in the brain is the general symptom of PA. This deficiency is caused by reduced activity of choline transferase (an acetylcholine forming enzyme) or increased acetylcholinesterase activity, resulting in a decrease the level of acetylcholine (Omar, 2010). Therefore, the dominant strategy used in the care of PA patients is aimed to increasing the potency of acetylcholine through reducing the degradation rate in the brain, it is achieved through providing a potent acetylcholinesterase (AChE) inhibitor (Liesener et al., 2007).

The compound discovery which has activity as AChE inhibitors can be an effective strategy for Alzheimer's disease treatment. Some compounds from natural

* Corresponding Author:
Nurul Jadid Mubarakati
Department of Biology, Faculty of Science and Mathematics,
University Islamic of Malang, Malang, Indonesia.
Phone: +6285791322022 Fax: +62341551356, 551822
e-mail: nurul.jadid@unisma.ac.id

ingredients have been used as medicines for Alzheimer disease including galantamine. In addition, various natural compounds from plants have been developed as AChE inhibitors, including polyphenols (Mendiola et al. 2016). Olea is one of a genus containing high polyphenols which has been found in Indonesia. Oleuropein, demethyl-oleuropein, ligstroside, verbascoside, luteolin, and oleoside are the main polyphenols of genus Olea. Several studies have shown that oleuropein compound isolated from olive can bind $\mathrm{AChE}$ and inhibit the interaction of AChE and amyloid-beta, thus prevent the formation of insoluble plaques (Ellis, 2005).

One of olea species which has been widely cultivated in Indonesia is Olea europaea. Nowadays, several natural products of olea have been circulating either in Indonesia market or abroad. One famous product which contains natural compound of Olive is Olive oil (known as extra virgin olive oil). According to previous research study (Ellis, 2005), Olive oil can overcome various diseases including Alzheimer's disease.

The metabolic fate of drug candidates has been explored and predicted through a number of computational approaches, allowing for screening of large numbers of chemical compounds and then identifying a small number of promising candidates (Kazmi et al., 2019). We introduce in silico approaches that have been developed to predict drug metabolism and fate, and assess their potential to facilitate the virtual discovery of promising drug candidates. We also provide a brief description of interaction model for predicting binding affinity of olive phenolic compound-AChE. 
The objective of this study is to explore the potential phenolic compounds of olive leaves that have AChE inhibitory potency through in silico approach as scientific support for Indonesia natural products. Furthermore, this study will provide information about potential compounds of olive for Alzheimer's disease treatment and increase scientifically the value added of olive product for sustainable welfare particularly in Indonesia.

\section{Methods}

\section{Target Identification}

The three-dimensional structure of acetylcholin esterase (AChE) was obtained from Protein Data Bank (PDB ID 4EY6).

\section{Selection of Ligands}

In this study, the use of ligand obtained from public repository PubChem included phenolic compounds of Olive leaves with their identification codes were identified as follow: Oleuropein (CID: 5281544), DemethylOleuropein (CID: 6450302), Ligstroside (CID: 14136859), Oleoside (CID: 101042548), Verbascoside (CID: 5281800), Luteolin 7-Glucoside (CID: 5280637), and Hydroxytyrosol (CID: 82755), and were selected as ligand. Those the three-dimensional (3D) polyphenols structure of Olive Compound used for docking analysis were formatted as SDF file.

\section{Bioactivity Prediction of Phenolic Compound in Olive leaves}

Biological activity prediction of phenolic content in olive leaves included oleuropein, demethyl-oleuropein, ligstroside, oleoside, verbascoside, luteolin 7-glucoside dan hydroxytyrosol were conducted using Way2Drug Pass Online (http://www.pharmaexpert.ru/passonline). The potential active compound of phenolic content in olive leaves were determined through comparison of similarity index (Pa Value) between their bioactive structure and potential recorded compound in the database by using Way2Drug PASS Online server (http://www.pharmaexpert.ru/passonline). The result of a potentially active compound was reviewed based on their $\mathrm{Pa}$ value described the potential compound that being tested. In addition, the three-dimensional (3D) structure is then constructed using the Discovery Studio Software (https://pubchem.ncbi.nlm.nih.gov/).

\section{Molecular Docking Analysis}

A computational ligand-target docking approach was applied to analyze complex structure of the AChE as target, with seven different polyphenols as ligands. The molecular docking analysis was run using AutoDock Vina with Pyrx Software. The active compound of galanthamine was used as a binding activity comparison between active compounds of olive plant and each 7 (seven) phenolic compound of Olive leaves. The docking analysis result are displayed binding affinity and ligandreceptor interaction.

\section{Results}

\section{The Bioactivity Prediction of Phenolic Compound in}

\section{Olive Leaves}

The biological activity of olive phenolic compound of oleuropein, demethyl-oleuropein, ligstroside, oleoside, verbascoside, luteolin 7-glucoside, and hydroxytyrosol included antioxidants, anti-inflammatory, antiatherogenic, anti-cancer/antineoplastic, anti-viral, skin protectant, anti-aging and neuroprotective activity of phenolic compound in Olive leaves were determined using Way2Drug Pass Online.

In general, the investigation result of Way2Drug Pass Online indicates that the phenolic compounds of Olive leaves contain bioactivity as antioxidant, antiinflammatory, antineoplastic, free radical scavenger, antibacterial, antifungal, enhancer for TP53, caspase 8 stimulant, inhibitor of platelet adhesion, treatment of lipoprotein disorder, antiviral, and dementia treatment. The highest bioactivity percentage of olive phenolic compound are as an antioxidant of $82 \%$, anti-inflammatory of $73 \%$, and anti-cancer (antineoplastic) of $70 \%$ as shown in Figure 1, respectively. Meanwhile, the lowest bioactivity percentage is as dementia treatment of $30 \%$. The antiinflammatory and antioxidant activity could be related to neuroinflammatory-related diseases, such as Alzheimer's disease. This study suggests that according to Way2Drug PASS Online analysis, the result elucidated that the role of phenolic compound in Olive leaves as an antioxidant and free radical scavenger is essential for anti-aging, neuroinflammatory, and skin protection.

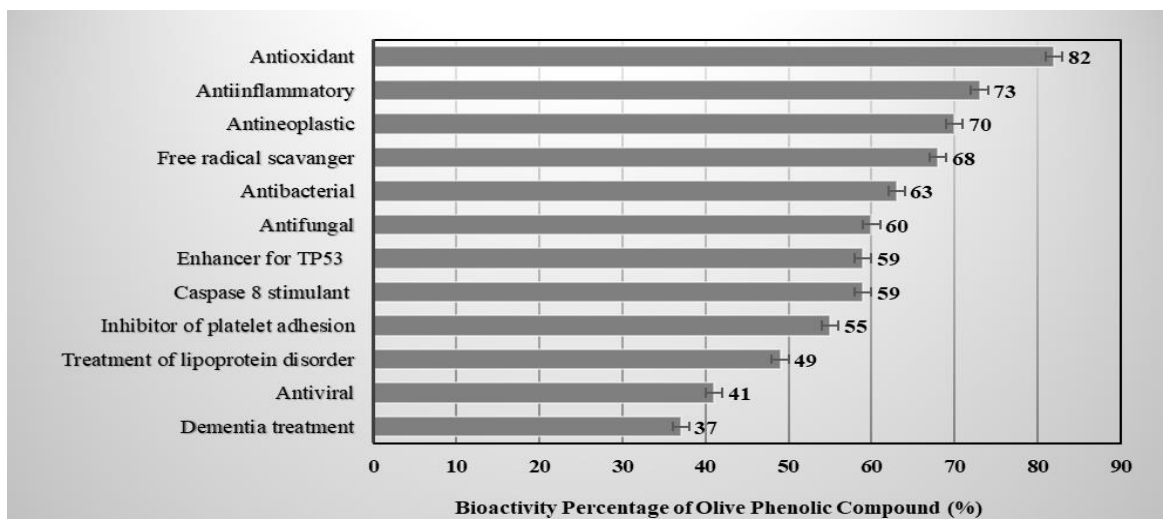

Figure 1. The bioactivity prediction of phenolic compound in olive leaves using Way2Drug PASS Online. 
Table 1. The binding affinity of olive phenolic compounds (ligand) with $\mathrm{AChE}$

\begin{tabular}{lc}
\hline $\begin{array}{l}\text { The phenolic compound of } \\
\text { olive leaves (Ligand) + AChE }\end{array}$ & Binding affinity (kcal/mol) \\
\hline Verbascoside + AchE & -2.8 \\
Luteolin 7-glucoside + AChE & -4.4 \\
Oleuropein + AChE & -5.2 \\
Hydroxytyrosol + AChE & -6.5 \\
Ligstroside + AChE & -7.2 \\
Oleoside + AChE & -8.8 \\
Demethyl-oleuropein + AChE & -8.9 \\
Galanthamine (Positive control) & -10.3 \\
\hline
\end{tabular}

The previous comprehensive studies of olive phenolic compound as antioxidant, anti-inflammatory and antineoplastic have been elucidated frequently that were indicated by the high bioactivity percentage of olive phenolic compound as antioxidant, anti-inflammatory and antineoplastic respectively (Fig. 1). Therefore, in this study we have not focused on the bioactivity analysis of antioxidant, anti-inflammatory and antineoplastic of each olive phenolic compound. Whereas, the discovery of acetylcholinesterase (AChE) inhibitor for dementia treatment such as Alzheimer disease is not well-known and becoming challenging area that were indicated by lowest bioactivity percentage of olive phenolic compound as dementia treatment (Fig. 1). In the following investigation we have outlined on the possible interaction between seven phenolic compounds of olive leaves with AChE of Alzheimer disease included binding affinity and structure analysis using AutoDock Vina with Pyrx Software (Tab. 1, Fig. 2 and 3).

\section{The Interactions between Phenolic Compound of Olive Leaves with Acetylcholinesterase (AChE)}

Nowadays, the natural medicine discovery of Alzheimer's disease is not well-known. Alzheimer's disease is caused by the loss of progressive cholinergic neurons and reduced acetylcholine in the brain which results in a decrease in intellectual ability or a decrease in cognitive function. In this study, the phenolic compounds of olive leaves were applied as a cholinesterase inhibitor which was considered to inhibit the activity of acetylcholinesterase in reducing acetylcholine after the neurotransmitter attached to the target cell receptor.

Seven active phenolic compounds of Olive leaves were analyzed for their binding affinity to $\mathrm{AChE}$ protein
(Tab. 1). Based on molecular docking analysis show that the binding affinity of Demethyl-oleuropein +AChE of $8.9 \mathrm{kcal} / \mathrm{mol}$ has closed to galanthamine binding affinity of $-10.3 \mathrm{kcal} / \mathrm{mol}$ (Tab. 1). The more negative binding affinity value obtained, the stronger the interaction occurred between each phenolic compound (ligand) and AChE. According to binding affinity value, demethyloleuropein is the most potential phenolic compound of olive leaves that could make it possible as an AChE inhibitor. Furthermore, the prediction of the binding model of demethyl-oleuropein at AchE active site was determined in Figure 2.

\begin{tabular}{|c|c|}
\hline Bioactive ligand & Interaction type \\
\hline Galanthamine & $\begin{array}{l}\text { Hydrogen bond: Glu202 } \\
\text { Hydrofobic bond: Asp74, Tyr124, Gly122, } \\
\text { Phe297, Phe338, Gly121, Ser125, Phe295, } \\
\text { Ser203, His447, Gly120, Trp86, Tyr337, } \\
\text { Asp74 }\end{array}$ \\
\hline $\begin{array}{l}\text { Demethyl- } \\
\text { oleuropein }\end{array}$ & $\begin{array}{l}\text { Hydrogen bond: Glu202, tyr133, Tyr } 124 \\
\text { Hydrofobic bond: Phe297, Gly122, His } 447 \text {, } \\
\text { Phe338, Gly120, Trp86, Gly121, Asp74, } \\
\text { Tyr341, Tyr337 }\end{array}$ \\
\hline
\end{tabular}

Molecular docking analysis shows that galanthamine molecules can bind to the active site of the AChE enzyme via hydrogen bonds with Glu202 amino acid residue, while demethyl-oleuropein has more than three hydrogen bonds with amino acid residues glu202, tyr133, tyr 124 (Tab. 2). The interaction type of dimethyl-oleuropein as a bioactive ligand with $\mathrm{AChE}$ are presented in Figure 3.

In this study, the galanthamine-AChE bond complex appears to be stabilized by hydrophobic interactions which involved amino acid residues Asp74, Tyr124, Gly122, Phe297, Phe338, Gly121, Ser125, Phe295, Glu202, Ser203, His447, Gly120, Trp86, Tyr337 and Asp74, whereas, demethyl-oleuropein-AChE bond complex was stabilized by hydrophobic interaction which included amino acid residues Phe297, Gly122, His447, Phe338, Glu202, Gly120, Tyr133, Trp86, Gly121, Asp74, Tyr341, Tyr337, Tyr124 (Fig. 3). The 3D model presented in this study indicates that demethyl-oleuropein of Olea europaea interact with AChE enzyme binding sites.

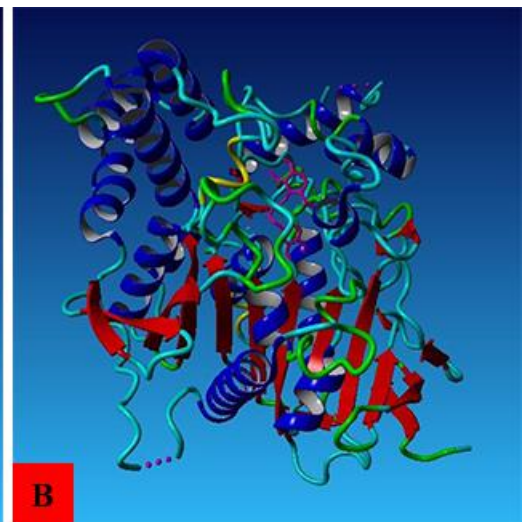

Figure 2. The 3D structure of interaction between galanthamine with AChE enzyme as a positive control (A); and interaction between demethyloleuropein with AChE (B). 


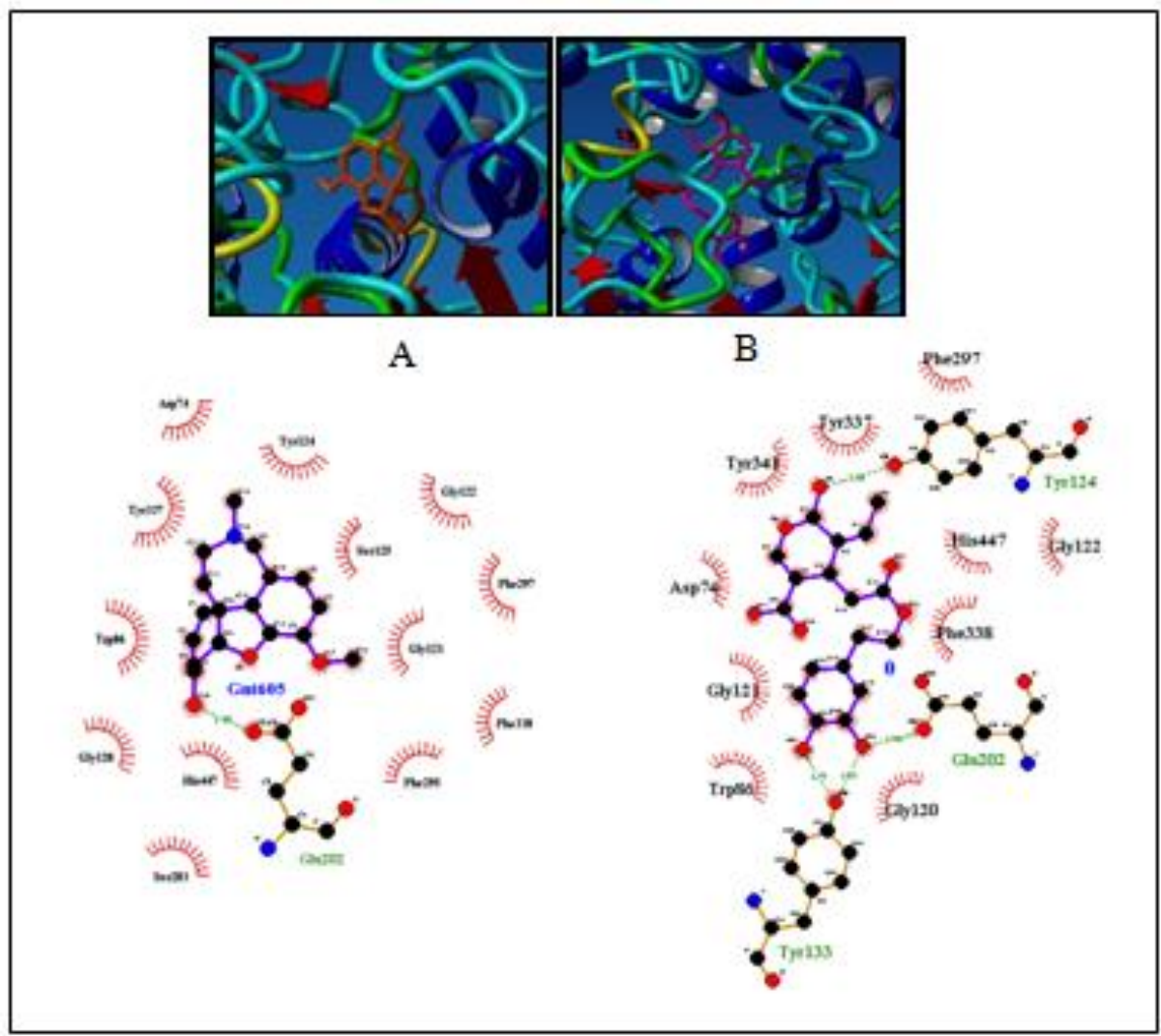

Figure 3. The 3D binding structure of bioactive ligand with AChE enzyme (Galanthamine (A); Demethyl-oleuropein (B))

\section{Discussion}

The Olive, Olea europaea contain phenolic compound of all plant parts. Oleuropein, demethyl - oleuropein, ligstroside, oleoside, verbascoside are phenolic compound that commonly found in fruit, while luteolin 7-glucoside and hydroxytyrosol in leave (Silva et al., 2006). According to previous studies, the pharmacological properties of olive oil and leaves have been recognized as important components of medicine and healthy diets due to their high phenolic content. The phenylpropanoids in Chinese olive showed remarkable anti-neuroinflammatory activity, as well as antioxidant and antidiabetic activities (Zhang et al., 2019). Based on Way2Drug PASS Online analysis, the phenolic content of olive leaves has high potential antioxidant, anti-inflammatory, anticancer and free radical scavenger, respectively (Fig. 1), these are relevant to those previous comprehensive studies.

The antioxidants are substances that inhibit oxidation and may prevent cell damage due to free radicals. Meanwhile, aging is a disease due to the accumulation of ROS (Reactive Oxygen Species/Free radical) which causes a decrease in function; this ROS will be neutralized through antioxidants and free radical scavenger. The presence of antioxidants and free radical scavenger will protect the body from the accumulation of ROS that can cause DNA damage (Silva et al., 2006). The role of olives as antiinflammatory also has an impact on the reduction of skin aging. The role of olives as neuroprotection can be represented by the function of olives as Free Radical Scavenger and also Lipoprotein Disorder Treatment.
Olives are mentioned to be neuroprotection because olives have activities to reduce LDL oxidation. While Olive as antineoplastic can be described more clearly through the ability of olives as caspase 8 stimulant and TP53 expression enhancer which will stimulate the occurrence of apoptosis. Olives act as anti-atherogenic due tu olives can inhibit the attachment of the plaque to the endothelium (platelet adhesion inhibitor). Those plaque formations occur due to oxidized lipids. Whereas, atherosclerosis is caused by platelet piles on the endothelial wall. This pile causes vasodilation and vasoconstriction to be disrupted, so that blood vessels does not constrict and supply of nutrients and oxygen optimally (Mendiola et al., 2016).

Moreover, the role of olive phenolic compound in the care of PA patients is aimed to increase the potency of acetylcholine through reducing the degradation rate in the brain. It is achieved through providing a potent AChE inhibitor (Liesener et al., 2007). The deficiency of cholinergic neurotransmission activity in the brain is caused by reduced activity of choline transferase (an acetylcholine forming enzyme) or increased acetylcholinesterase activity, resulting in a decrease the level of acetylcholine (Omar, 2010).

As one of olive phenolic compounds, dimethyloleuropein could interact with AChE (Fig. 2B). Intermolecular interactions between dimethyl-oleuropein molecules with AChE enzyme as amino acid residues are indicated with complementary hydrogen, hydrophobic 
and electrostatic bond (Tab. 2). Hydrophobic interactions between non-polar side chains of amino acid residues are the main reason for protein fold in and interaction to form distinctive structures (Fig. 2 and 3). The hydrophobic interactions cause most of the hydrophobic amino acid side chains to be pushed into the interior of the protein to protect it from water (Murray et al., 2009). Moreover, alkaloids can bind to several binding sites and form intermolecular interaction via hydrogen bonds, hydrophobic nteractions, pp interactions, and hydrophilichydrophobic interactions and can also inhibit AChE activity, although with a lower potential.

Related to the previous research (Choudhary et al., 2005), this study also reveals the interaction between demethyl-oleuropein with AChE enzyme that may form a stable enzyme complex through intermolecular hydrogen bonds and pi-stacking interactions may further inhibit the activity of cholinesterase in cleavage of acetylcholine (Fig. 2). Those decreasing in acetylcholinesterase, an increasing of acetylcholine is expected (Ellis, 2005; Nugroho et al., 2017).

In this study, phytochemicals, phenolic compound of olive leaves, used as neuroprotectant. These phytochemicals can effectively reverse the age-related decline in cognitive function by inducing the expression of neurotrophins via the Trk signaling pathway in the hippocampus (Spencer et al., 2009). Neurotrophin binding triggers the activation of the Trk receptor, causing oligomerization and transautophosphorylation in intracellular domain, which leads to the activation of an intracellular signaling pathway with activation of Ras/mitogen activated protein kinase (MAPK), which results in CREB-dependent neurotrophin secretion and

\section{Acknowledgment}

The authors gratefully acknowledge University Islamic of Malang for financial support under the Research University Grant (RUG) in 2018. The author would like to thank Didik Huswo Utomo for providing a database of active compounds.

\section{References}

Carito, V., Venditti, A., Bianco, A., Ceccanti, M., Serrilli, A. M., Chaldakov, G., Tarani, L., de Nicolò, S., \& Fiore, M. (2014). Effects of olive leaf polyphenols on male mouse brain NGF, BDNF and their receptors trkA, trkB and p75. Natural Product Research, 28(22), 1970-1984.

Choudhary, M. I., Nawaz, S. A., Zaheer-ul-Haq, Azim, M. K., Ghayur, M. N., Lodhi, M. A., Jalil, S., Khalid, A., Ahmed, A., Rode, B. M., Atta-ur-Rahman, Gilani, A. U., \& Ahmad, V.U. (2005) Juliflorine: a potent natural peripheral anionic site-binding inhibitor of acetylcholinesterase with calcium-channel blocking potential, a leading candidate for Alzheimer's disease therapy. Biochemical and Biophysical Research Communications, 332, 1171-1177.

Descamps, S., Toillon, R. A., Adriaenssens, E., Pawlowski, V., Cool, S. M. (2001). Nerve growth factor stimulates proliferation and survival of human breast cancer cells through two distinct signaling pathways. Journal of Biology Chemistry, 276(21), 17864-17870.
Bcl-2 expression, promoting survival, proliferation and differentiation of the cell (Descamps et al., 2001).

In addition to their special biological activities, phytochemicals mainly act as antioxidants, scavenging free radicals in the brain and thus induces neuronal regeneration, and neuroprotection activities that lead to improved neuronal survival, differentiation, LTP, and memory enhancement (Gupta \& Sharma, 2017). Another studies showed that olive polyphenols may not only elicit an activation of the rodent olfactory system by increasing the levels of NGF and BDNF but also be stressing for the animal by reducing both the levels of hippocampal NGF/BDNF and serum GSH and increasing serum levels of NGF and BDNF (Carito et al., 2014). NGF and BDNF were neurotrophins that were identified as neuronal survival promoting proteins in mammals. A decrease in neurotrophins has been associated with the pathology of several neurodegenerative diseases and their physiological symptoms (Gupta \& Sharma, 2017).

Based on this finding of exploration of the phenolic compound of olive leaves as AChE inhibitor through in silico approach can be concluded that the dimethyloleuropein is the most potential phenolic compound of olive leaves that could be applied as AChE inhibitor for further possible drug candidate of degenerative disease therapy such as Alzheimer (Choudhary et al., 2005). The olive phenolic compound with AChE inhibitor activity may improve neurodegenerative diseases. In this study, the olive phenolic compound showed remarkable antioxidant activity, as well as anti-inflammatory and antineoplastic activities (Fig. 1). This suggests that Olive could be served as a natural health fruit, which was expected to be suitable for application as supplement with beneficial effects against neuroinflammatory-related diseases, such as Alzheimer's disease.

Ellis, J. (2005). Cholinesterase inhibitor in the treatment of dementia. The Journal of the American Osteopathic Association, 105, 145148.

Gupta, V. K., \& Sharma, B. (2017). Role of phytochemicals in neurotrophins mediated regulation of Alzheimer's disease. Internationa Journal of Complementary \& Alternative Medicine, 7(4), 00231

Kazmi, S.R., Jun, R., Yu, M. S., Jung, C., \& Na, D. (2019). In silico approaches and tools for the prediction of drug metabolism and fate: a review. Computer in Biology and Medicine, 106, 54-64.

Konrath, E. L., Passos, C. D. S., Klein-Júnior, L. C., * Amélia, T. (2013). Alkaloids as a source of potential anticholinesterase inhibitors for the treatment of Alzheimer's disease. Henriques Journal of Pharmacy and Pharmacology, 65, 1701-1725.

Liesener, A., Perchuc, A. M., Schöni, R., Schebb, N. H., Wilmer, M., \& Karst, U. (2007). Screening of acetylcholinesterase inhibitors in snake venom by electrospray mass spectrometry. Pure and Applied Chemistry, 79, 2339-2349.

Mendiola-Precoma, J., Berumen, L. C., Padilla, K., \& Garcia-Alcocer, G. (2016). Review article: therapies for prevention and treatment of Alzheimer's disease. BioMed Research International, 2016, 17.

Murray, R. K., Granner, D. K., \& Rodwell, V. W. (2009). Harper Biochemistry. Jakarta: EGC. (in Indonesian).

Nugroho, A., Choi, J. S., Hong, J. P., \& Park, H. J. (2017). Antiacetylcholinesterase activity of the aglycones of phenolic glycosides isolated from Leonurus japonicas. Asian Pacific Journal of Tropical Biomedicine, 7, 849-854. 
Omar, S. H. (2010). Review open access oleuropein in olive and its pharmacological effects. Scientia Pharmaceutica, 78, 133-154.

Silva, S., Gomes, L., Leitão, F., Coelho, A. V., \& Vilas, B. L. (2006). Phenolic compounds and antioxidant activity of Olea europaea L. fruits and leaves. Food Science and Technology International, 12, 385-396.

Spencer, J. P. E., Vauzour, D., \& Rendeiro, C. (2009). Flavonoids and cognition: the molecular mechanisms underlying their behavioural effects. Archives of Biochemistry and Biophysics, 492(1-2), 1-9.

Zhang, S., Huang, Y., Li, Y., Wang, Y., \& He, X. (2019). Antineuroinflammatory and antioxidant phenylpropanoids from Chinese olive. Food Chemistry, 286, 421-427. 